\title{
EFEITO DA BANDAGEM ELÁSTICA NO POSICIONAMENTO DA PELVE COM INCLINAÇÃO POSTERIOR
}

\author{
EFFECT OF THE ELASTIC BANDAGE ON THE POSITIONING OF THE PELVIS WITH POSTERIOR TILT \\ EFECTO DEL VENDAJE NEUROMUSCULARENEL POSICIONAMIENTO DE LA PELVIS CON \\ INCLINACIÓN POSTERIOR
}

Giulia Farina Lenzi' (Fisioterapeuta)

Tobias Jacoby ${ }^{\top}$ (Fisioterapeuta)

Gustavo Portella' (Fisioterapeuta)

Fabiana Silva' (Fisioterapeuta)

1. Centro Universitário Metodista, Instituto Porto Alegre (IPA), Curso de Fisioterapia, Porto Alegre, RS, Brasil.

\section{Correspondência:}

Avenida Mariland, 1027, apto. 401, Auxiliadora, Porto Alegre, RS, Brasil. 90440-191.

giulia.farina@gmail.com

\section{RESUMO}

Introdução: A inclinação posterior da pelve (IPP) pode levar à perda da curvatura lombar fisiológica normal, predispondo o indivíduo a lesões. A bandagem elástica (BE) tem sido utilizada em disfunções musculoesqueléticas, podendo fornecer aos músculos um feedback para manter e perceber o alinhamento postural adequado. Não foram encontrados estudos sobre a influência do uso da bandagem na IPP. Objetivo: Avaliar o efeito da BE na ativação do músculo tensor da fáscia lata em indivíduos com IPP. Métodos: Trata-se de um ensaio clínico randomizado em que foi realizada uma avaliação do posicionamento da pelve de 20 homens com idade entre 18 e 28 anos. Os indivíduos foram divididos em dois grupos de forma aleatória; no grupo bandagem (GB), a bandagem foi aplicada com tensionamento no músculo tensor da fáscia lata e do trato iliotibial, e no grupo placebo (GP) foi aplicado o mesmo material, porém sem tensionamento, por um período de 72 horas. Os grupos foram reavaliados 30 minutos e 72 horas após a intervenção. Resultados: Houve diminuição significativa da IPP no GB $(p=0,002)$ quando comparado com o GP $(p=0,146)$. A resposta mais significativa da inclinação no GB foi observada no período imediato em comparação com 72 horas depois. Conclusão: Os achados deste estudo demonstram que a técnica da BE pode auxiliar na diminuição da IPP. São necessários mais estudos que avaliem a ativação muscular e a repercussão do uso da BE em indivíduos com IPP.

Descritores: pelve; bandagens; força muscular; fascia lata.

\section{ABSTRACT}

Introduction: The posterior pelvic tilt (PPT) may lead to loss of the normal physiologic lumbar curve, predisposing the subject to injury. Elastic bandage (EB) has been used in musculoskeletal disorders and can provide feedback to the muscles, enabling them to perceive and keep adequate postural alignment. No studies were found on the influence of elastic bandage use in PPT. Objective: To evaluate the effect of EB on the muscular activation on the tensor fasciae latae muscle in subjects with PPT. Methods: This was a randomized clinical trial in which the pelvis positioning of 20 men aged 18 to 28 years was evaluated The subjects were randomly divided into two groups, the bandage group (BG) in which the EB was applied tensioning the tensor fasciae latae muscle and the iliotibial tract, and the placebo group (PG), with the same material applied to subjects, but without tension, for a period of 72 hours. The groups were reassessed 30 minutes and 72 hours after the intervention. Results: There was a significant decrease in PPT in the BG $(p=0.002)$ compared to the $P G(p=0.146)$. The largest inclination reduction in the $B G$ was observed in the immediate period compared to 72 hours later. Conclusion: The findings of this demonstrate that the EB technique can help the reduction of PPT. Further studies are needed to evaluate the muscle activation and the repercussions of the use of EB in subjects with PPT.

Keywords: pelvis; bandages; muscle strength; fascia lata.

\section{RESUMEN}

Introducción:La inclinación pélvica posterior (IPP) puedellevar a la pérdida de la curvatura lumbar fisiológica normal, lo que predispone el individuo a una lesión. El vendaje neuromuscular (VN) se ha utilizado en los trastornos musculoesqueléticos y puede proporcionar feedback a los músculos para que mantengan y perciban la alineación postural adecuada. No se han encontrado estudios sobre la influencia del uso de vendaje sobre la IPP. Objetivo: Evaluar el efecto del VN en la activación del músculo tensor de la fascia lata en individuos con IPP. Métodos: Se trata de un ensayo clínico aleatorio en el cual se llevó a cabo una evaluación de la posición de la pelvis de 20 hombres de edades comprendidas entre los 18 y los 28 años. Se ha dividido los individuos en dos grupos de forma aleatoria; en el grupo de vendaje (GV), el vendaje se aplicó con tensión en el músculo tensor de la fascia lata y el tracto iliotibial, y en el grupo placebo (GP) se aplicó el mismo material, pero sin tensión, durante un período de 72 horas. Los grupos se evaluaron 30 minutos y 72 horas después de la intervención. Resultados: Se observó una disminución significativa de la IPP en GV $(p=0,002)$ en comparación con el GP $(p=0,146)$. La respuesta más significativa de la inclinación en el GV se observó en el período inmediato en comparación con las 72 horas. Conclusión: Los resultados de este estudio muestran que la técnica del VN puede ayudar a reducir la IPP. Se necesitan más estudios para evaluar la activación muscular y el efecto de la utilización del VN en pacientes con IPP.

Descriptores: pelvis; vendajes; fuerza muscular; fascia lata. 


\section{INTRODUÇÃO}

O alinhamento e controle da pelve e do tronco são necessários para fornecer a base estável sobre a qual os membros podem se movimentar, principalmente durante a marcha. O desalinhamento da pelve ocasiona sobrecarga indevida sobre o sistema musculoesquelético'. Dentre as alterações pélvicas, destaca-se a inclinação posterior (IPP). A IPP ocorre quando o plano vertical através das espinhas ilíacas ântero-superiores (EIAS) é posterior ao plano vertical da sínfise púbica². Essa alteração pode levar a perda da curvatura lombar fisiológica normal, aumentando o estresse intervertebral ${ }^{2} \mathrm{e}$ predispondo o indivíduo a lesões na coluna ${ }^{3}$. É observado um desequilíbrio em toda cadeia muscular que envolve a pelve, apresentando os músculos flexores de tronco e extensores do quadril retraídos, e seus antagonistas, alongados e enfraquecidos?.

A bandagem elástica tem sido utilizada em disfunções musculoesqueléticas e neuromusculares, promovendo benefícios para o sistema sensoriomotor e a propriocepção; além disso, pode inibir ou ativar musculaturas, reduzir inflamações e diminuir a dor $r^{4,5}$. A bandagem funcional fornece para os músculos um feedback para manter o alinhamento postural adequado ${ }^{6}$. Porém, são escassos os estudos que avaliam os efeitos da bandagem funcional, principalmente envolvendo a posição da pelve, ainda que clinicamente seja uma técnica bastante utilizada. A sua aplicação pode contribuir para o meio terapêutico na busca do alinhamento pélvico, o que pode minimizar danos ao sistema musculoesquelético. Por estes motivos, o objetivo do presente estudo é avaliar o efeito da bandagem elástica na ativação muscular do músculo tensor da fáscia lata em indivíduos com inclinação posterior da pelve.

\section{MATERIAIS E MÉTODOS}

Esse estudo foi submetido e aprovado pelo Comitê de Ética em Pesquisa do Centro Universitário Metodista - IPA, sob o protocolo de no 334/2011, em 19/01/2012. Todos os participantes assinaram o Termo de Consentimento Livre e Esclarecido, em duas vias, autorizando sua participação. Trata-se de um ensaio clínico randomizado controlado com cegamento. No estudo foram incluídos 20 voluntários que apresentavam IPP, com faixa etária de 18 a 28 anos de idade e que estivessem pelo menos há uma semana sem realizar atividade física. Após a inclusão no estudo, foi realizado um sorteio, de forma aleatória, através do uso de envelopes pardos, a fim de definir a que grupo cada indivíduo pertenceria, sendo um o grupo bandagem (GB) e outro o grupo placebo (GP), ambos com 10 indivíduos cada. Visualmente não havia diferença entre os grupos, pois em ambos foi aplicada a bandagem, diferindo apenas o tensionamento, que reforçava o cegamento do avaliador.

A coleta foi realizada no Laboratório do Movimento do Centro Universitário Metodista - IPA. A avaliação da pelve foi realizada por um avaliador treinado, cegado em relação à qual grupo os indivíduos pertenciam. A avaliação do posicionamento da pelve seguiu o modelo proposto por Santos ${ }^{7}$. O indivíduo posicionou-se em pé, mantendo os pés em posição de passo. O avaliador marcou inicialmente um ponto na espinha ilíaca póstero-inferior (EIPI). Após, localizou a espinha ilíaca ântero-superior (EIAS) e marcou outro ponto. Localizadas as marcas anatômicas, foi traçada uma linha de cada ponto marcado até a linha axilar do indivíduo. Caso as linhas não se encontrassem, era medida a distância, no sentido vertical, entre elas com uma régua. Foi considerado dentro da normalidade, para o sexo masculino, até $1 \mathrm{~cm}$ de inclinação posterior, sendo este um critério de exclusão (Figura 1)

A aplicação da bandagem foi realizada pelo pesquisador do estudo. Antes da aplicação, os pelos da região foram aparados com um cortador de cabelo da marca sOlac ${ }^{\oplus}$ (Vitoria, Alava, Espanha), seguido de assepsia com algodão banhado em álcool 70\% no local onde foi aplicada a bandagem elástica da marca 3NS TEX® (Seul, Gangbuk-gu, Coreia do Sul).

No GB, foi aplicada a bandagem de $5 \mathrm{~cm}$ de largura, em formato I, sem cortes, com tensão de $50 \%$ sobre o músculo tensor da fáscia lata, no sentido da origem (EIAS) à inserção (trato ílio tibial) com a musculatura em alongamento. $\mathrm{O}$ alongamento foi realizado com o indivíduo deitado em decúbito lateral com a perna a ser alongada para cima, e o pesquisador posicionou o membro em extensão e adução de quadril. As pontas das extremidades da bandagem foram cortadas de forma que ficassem em formato de " $C$ ", para que não houvesse risco de descolamento. O GP recebeu a aplicação com o mesmo material, na mesma localização e formato, porém a bandagem realizada sem tensão e sem alongamento da musculatura. (Figura 2)

Após a aplicação, o indivíduo foi orientado a retornar 30 minutos depois, sendo livre qualquer posição ao indivíduo. Foi enfatizado de que não deveriam praticar atividade física durante a realização do estudo. Os indivíduos realizaram atividades cotidianas, o que condiz com a prática clínica em geral. Após o tempo estipulado, foi realizada uma nova avaliação da pelve com a bandagem. Ao término desta avaliação, o participante foi convidado a retornar no período de 72 horas. Passado o intervalo, a bandagem foi retirada e foi realizada a última avaliação para comparação dos resultados obtidos nas medidas da inclinação da pelve, entre o pré, pós-imediato e pós-72h.

\section{Análise estatística}

A análise dos dados foi realizada utilizando o software Statistical Package for the Social Sciences (SPSS), versão 18.0. As variáveis contínuas foram descritas por média e desvio padrão, e as variáveis categóricas, por frequências absolutas e relativas. Na comparação de variáveis contínuas entre os grupos, o teste t-Student para amostras independentes foi aplicado. Para as variáveis categóricas, o teste exato de Fisher foi utilizado. Para comparar os lados direito e esquerdo, em ambos os grupos, o teste t-Student para

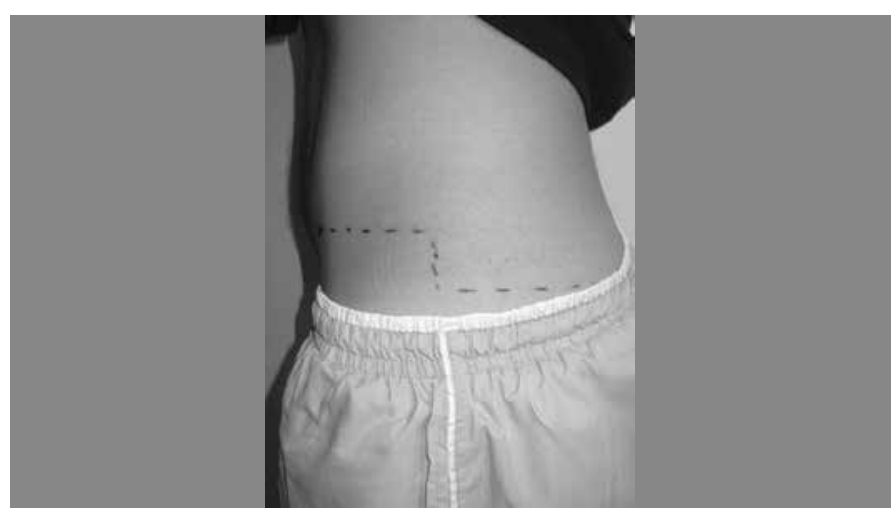

Figura 1. Avaliação postural.

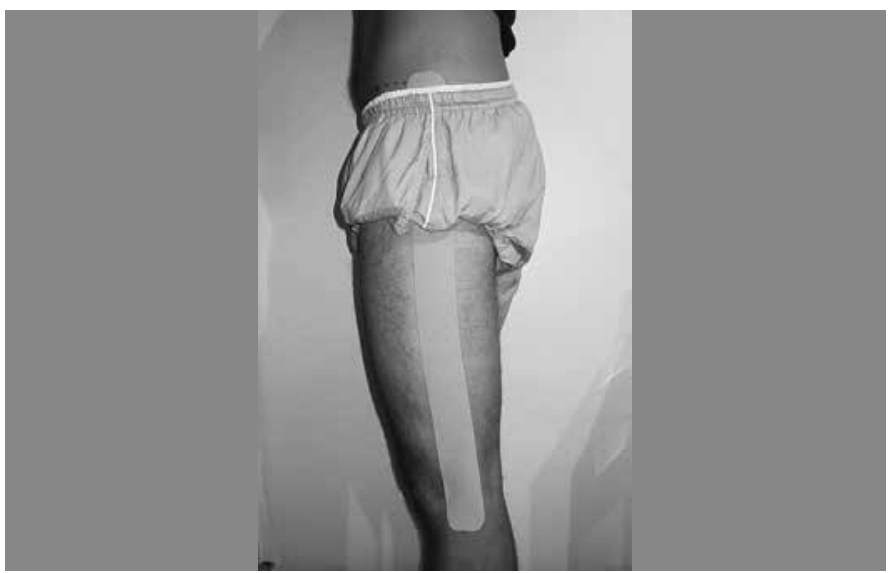

Figura 2. Bandagem Funcional. 
amostras pareadas foi aplicado. Para comparar a inclinação posterior da pelve ao longo das três medições, conforme grupo, a Análise de Variância (ANOVA) two-way para medidas repetidas foi aplicada. Para complementar essa análise, o teste de Bonferroni foi utilizado.

O nível de significância estatística considerada foi de 5\% ( $p>0,05)$.

\section{RESULTADOS}

A amostra deste estudo foi constituída por 20 participantes, 10 no GB e 10 no GP, com média de idade de 24,2 \pm 2,62, no GB, e 21,3 \pm 2,95, no GP. Quanto à prática de atividade física, apenas cinco indivíduos no GB e seis no GP alegaram realizar regularmente; apenas um voluntário do GB apresentou queixas álgicas na região lombar durante o período do estudo (Tabela 1).

No GB houve diminuição significativa da IPP em relação ao GP, conforme demonstra a Tabela 2. A maior diminuição foi observada no período que se estendeu da pré-aplicação ao pós-imediato. No período do pós-imediato ao pós-72h, os valores se mantiveram semelhantes na análise dos grupos. Na análise do GB, o lado direito no pré-aplicação diferiu do pós-imediato e do pós-72h; já no lado esquerdo, o préaplicação e o pós-imediato diferiram do pós-72h. No GP não houve diferença significativa, tanto no lado direito quanto no esquerdo.

No entanto, é importante destacar que o GB inicia com valores significativamente mais altos do que o GP. Desta forma, houve um ajuste dos níveis basais e da idade, e a diferença permaneceu significativa $(p=0,023)$. A utilização desta medida é válida, visto que não houve diferença significativa entre os valores dos lados direito e esquerdo ( $p>0$, 300) em ambos os grupos.

A média da IPP nos dois grupos está descrita na Figura 3.

Tabela 1. Caracterização da amostra.

\begin{tabular}{c|c|c|c}
\hline Variáveis & $\begin{array}{c}\text { Grupo } \\
\text { bandagem } \\
(\mathbf{n = 1 0 )}\end{array}$ & $\begin{array}{c}\text { Grupo } \\
\text { placebo } \\
(\mathbf{n}=\mathbf{1 0})\end{array}$ & $\mathbf{p ~}$ \\
\hline Idade (anos) - média \pm desvio padrão & $24,2 \pm 2,62$ & $21,3 \pm 2,95$ & 0,032 \\
\hline Altura (m) - média \pm desvio padrão & $1,77 \pm 0,07$ & $1,75 \pm 0,08$ & 0,499 \\
\hline Atividade física $-\mathrm{n}(\%)$ & & & 1,000 \\
\hline Sim & $5(50,0)$ & $6(60,0)$ & \\
\hline Não & $5(50,0)$ & $4(40,0)$ & \\
\hline Quadro álgico & & & 1,000 \\
\hline Sim & $1(10,0)$ & $0(0,0)$ & \\
\hline Não & $9(90,0)$ & $10(100)$ & \\
\hline
\end{tabular}

Tabela 2. Comparação da inclinação da pelve, em cm, ao longo do tempo conforme o grupo.

\begin{tabular}{|c|c|c|c|}
\hline Variáveis & $\begin{array}{c}\text { Grupo bandagem }(n=10) \\
\text { Média } \pm \text { DP }\end{array}$ & $\begin{array}{c}\text { Grupo placebo }(n=10) \\
\text { Média } \pm \text { DP }\end{array}$ & $p^{*}$ \\
\hline \multicolumn{4}{|l|}{ Lado direito } \\
\hline Pré & $3,36 \pm 1,10^{b}$ & $2,47 \pm 0,71$ & 0,046 \\
\hline Pós-imediato & $2,75 \pm 1,01^{\mathrm{a}}$ & $2,43 \pm 0,72$ & 0,424 \\
\hline Pós 72h & $2,40 \pm 1,10^{\mathrm{a}}$ & $2,38 \pm 0,64$ & 0,961 \\
\hline$p^{* *}$ & 0,001 & 0,160 & \\
\hline \multicolumn{4}{|l|}{ Lado esquerdo } \\
\hline Pré & $3,52 \pm 0,92^{b}$ & $2,27 \pm 0,63$ & 0,002 \\
\hline Pós-imediato & $2,93 \pm 1,03^{b}$ & $2,28 \pm 0,70$ & 0,116 \\
\hline Pós 72h & $2,49 \pm 0,97^{a}$ & $2,23 \pm 0,61$ & 0,482 \\
\hline$p^{* *}$ & 0,004 & 0,266 & \\
\hline \multicolumn{4}{|l|}{ Geral } \\
\hline Pré & $3,44 \pm 0,91^{b}$ & $2,37 \pm 0,59$ & 0,006 \\
\hline Pós-imediato & $2,84 \pm 0,84^{\mathrm{a}}$ & $2,36 \pm 0,66$ & 0,170 \\
\hline Pós 72h & $2,45 \pm 0,95^{\mathrm{a}}$ & $2,31 \pm 0,57$ & 0,695 \\
\hline$p^{* *}$ & 0,002 & 0,146 & \\
\hline
\end{tabular}

\section{DISCUSSÃO}

A pelve é considerada uma das estruturas chaves no alinhamento das estruturas corporais. Qualquer alteração em sua posição poderá causar movimentos compensatórios em outras partes do corpo, sendo a coluna lombar e o quadril os primeiros a serem prejudicados ${ }^{1,8}$. Uma alteração importante na posição da pelve é a inclinação posterior que ocorre quando a espinha ilíaca ântero-superior se encontra mais cefálica em relação à espinha ilíaca póstero-inferior ${ }^{7}$ Em relação à avaliação do posicionamento da pelve, foi utilizada a proposta por Santos, sendo um método barato, simples e rápido de se mensurar possíveis desvios posturais no indivíduo ${ }^{9}$. Esta medida clínica possui Coeficiente de Correlação (ICC) de 0,71 para detecção de inclinação posterior com $p=0,01$, verificada intra-avaliadores, sendo um total de três avaliadores em três momentos diferentes, conforme os achados de Pinheiro et al. ${ }^{9}$. A IPP pode ser uma consequência do tensionamento dos músculos isquiostibiais, reto abdominal, períneo, glúteo máximo e as fibras posteriores do glúteo médio e do alongamento e/ou enfraquecimento dos músculos paravertebrais, reto femoral e da fáscia toracolombar ${ }^{10}$. O músculo tensor da fáscia lata, quando atua bilateralmente na pelve exercendo tensão, produz uma inclinação anterior e, ao atuar em conjunto com a musculatura envolvida na pelve, colabora para a manutenção do equilíbrio da mesma. Neste estudo, foi utilizada a aplicação da bandagem elástica no músculo tensor da fáscia lata, bilateralmente, por este ser mais superficial e estar em maior contato com a fáscia, tornando a aplicação da bandagem mais eficaz, o que potencializaria a ação do músculo na inclinação anterior da pelve.

No presente estudo, foi observada uma diminuição significativa da IPP no GB. Acredita-se que a aplicação da bandagem facilitou a ação muscular através de uma tração concêntrica da fáscia muscular. $\mathrm{O}$ alinhamento das fibras musculares pode ter contribuído para o reposicionamento da pelve, pois no GB houve tensionamento de 50\% da bandagem elástica ${ }^{11}$. Outros estudos sugerem que os efeitos da bandagem podem ocorrer por aumento do recrutamento de unidades motoras do músculo ${ }^{12,13}$. Entretanto, Halseth et al. ${ }^{6}$ acreditam que a aplicação da bandagem elástica gera uma estimulação sensorial, o que aumenta a entrada proprioceptiva, reforçando assim o controle postural. Nesta perspectiva, a estimulação poderia ter ocorrido no GP, no qual também foi aplicada a bandagem, porém sem tensão, mas neste grupo não houve diferença significativa na inclinação da pelve.

Esses efeitos também têm sido estudados através da utilização da eletromiografia para verificar se a bandagem elástica possui o efeito de ativação muscular ${ }^{14}$. Slupik et al. ${ }^{12}$ demonstraram em seu estudo que a aplicação da bandagem promoveu uma modificação da atividade bioelétrica do músculo vasto medial durante a contração isométrica

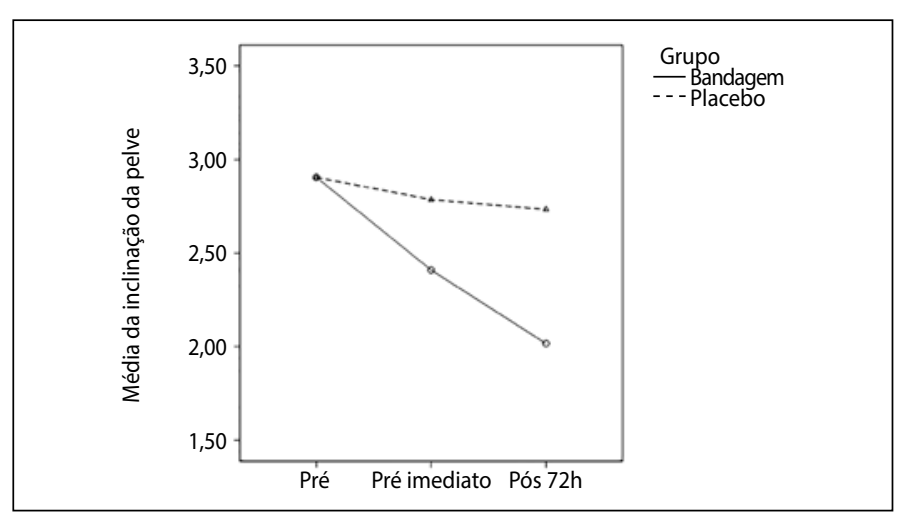

Figura 3. Média da inclinação da pelve. 
do quadríceps, por um aumento no recrutamento de unidades motoras. Corroborando, no estudo de Chen et al. ${ }^{15}$, foram recrutados indivíduos com síndrome femoropatelar que receberam aplicação da bandagem no músculo vasto medial e indivíduos saudáveis que receberam a mesma forma de aplicação, como placebo, e foi verificado um aumento na velocidade de ativação do músculo vasto medial nos indivíduos que receberam a bandagem. Ao encontro deste estudo, Hsu et al. ${ }^{16}$ investigaram os efeitos da bandagem na cinemática, ativação muscular e força da região escapular, estimulando o músculo trapézio superior, o que proporcionou um melhor posicionamento escapular, ocasionando uma redução da compressão causadora do impacto no ombro.

Alexander et al. ${ }^{17}$ inferiram que a ativação muscular ocorre devido a um aumento de unidades motoras recrutadas através do estímulo proprioceptivo que a bandagem causa. No entanto, esta proposição foi refutada por algumas publicações, que mostraram não haver aumento significativo na atividade eletromiográfica após a utilização da bandagem ${ }^{18}$. Corroborando, Janwantanakul e Gaogasigam ${ }^{19}$ avaliaram o efeito das técnicas da bandagem para facilitação e inibição da atividade dos músculos vasto lateral e vasto medial oblíquo em mulheres saudáveis, comparando os resultados com o grupo controle, e verificaram que não houve diferença significativa entre os indivíduos com bandagem e sem bandagem na atividade eletromiográfica destes músculos. Fu et al. ${ }^{20}$ também não observaram nenhuma melhora nas medidas isocinéticas ou inibição do torque muscular nos extensores ou flexores de joelho quando a bandagem foi aplicada no músculo quadríceps em atletas saudáveis. No presente estudo não foi utilizada a avaliação eletromiográfica para verificar a ativação muscular.

Existem controvérsias em relação à função da bandagem elástica na ativação muscular, mas, de acordo com os resultados encontrados neste estudo, acredita-se que a bandagem pode ter acompanhado o movimento do músculo tensor da fáscia lata durante a marcha, e isso pode ter gerado um estímulo exteroceptivo, fornecendo um feedback postural para a diminuição da inclinação da pelve. Este estímulo sensorial pode ter aumentado a entrada proprioceptiva, contribuindo para o melhor controle postural da pelve.

A bandagem cria uma pressão e estira a pele. Ao estimular os mecanorreceptores cutâneos 21,22 , este carregamento externo provoca alterações fisiológicas na região onde ocorreu a aplicação22. Os mecanorreceptores são principalmente encontrados na pele e respondem ao tato e deformações da mesma. Sendo assim, a informação mecanorreceptora se une à informação do fuso muscular e dos receptores cutâneos para informar o ângulo articular ${ }^{23}$. Os estímulos mecânicos são percebidos por uma enorme variedade de neurônios especializados com corpos celulares localizados nos gânglios espinais e cranianos, ramificando-se até a derme e a fáscia superficial ${ }^{24}$. Os receptores articulares, musculares e cutâneos contribuem para a sensação de movimento, enquanto a sensação de posição do corpo ou de parte dele é primordialmente sinalizada pelos receptores cutâneos de adaptação lenta e pelo fuso muscular ${ }^{25}$.

O tensionamento e a forma de aplicação da bandagem utilizados no presente estudo seguiu os preceitos de Kase et al. ${ }^{26}$, em que, para musculaturas fracas e/ou ativação de um músculo, a bandagem deve ser aplicada no sentido origem-inserção do músculo, pois essa posição facilitaria a função muscular. Morrissey ${ }^{27}$ reforça que, quando a bandagem é aplicada na direção das fibras musculares, a força do músculo subjacente pode ser facilitada. Neste caso, para a ativação muscular, a tensão da bandagem deve variar entre $50-75 \%$ para que ocorra percepção dos mecanorreceptores ${ }^{26}$

A ativação muscular ocorre entre 24 e 48 horas após a aplicação, e pode permanecer por até 72 horas após a retirada da bandagem ${ }^{12}$, caracterizando uma resposta tardia. Contribuindo, Karatas et al. ${ }^{28} \mathrm{citam}$ que o efeito fisiológico da bandagem é normalmente visto 72 horas após a aplicação. Entretanto, em um estudo de Lee e Yoo ${ }^{29}$ que utilizou a bandagem funcional para buscar a inclinação anterior da pelve, foi verificado efeito imediato após, ocorrido apenas com 30 minutos de intervenção, corroborando com os achados encontrados no presente estudo. Porém, houve pouca alteração nos achados no período do pré-imediato ao pós-72h. Isso pode ter ocorrido pela utilização prolongada da bandagem, o que pode fazer com que suas propriedades elásticas se percam devido ao atrito com roupas, banho e sudorese.

Embora alguns autores relatem melhora da dor e função através do uso da bandagem elástica ${ }^{26-28}$, uma série de revisões sistemáticas atuais tem demonstrado que a diminuição da dor pode estar relacionada a um efeito placebo ${ }^{30-32}$. No presente estudo, um indivíduo teve queixa álgica na região lombar no período em que permaneceu com a bandagem, sendo esta resolvida no momento da sua retirada. Isto pode ter ocorrido pela alteração causada na posição da pelve, já que este indivíduo pertencia ao GB. A pelve representa um elemento de transmissão de pressões devidas ao peso do corpo e pressões opostas advindas do solo através dos membros inferiores ${ }^{10}$. Sabe-se também que existe uma interligação miofascial entre a região lombar e a pelve ${ }^{7}$ e que a tensão da bandagem pode ter refletido no posicionamento da coluna lombar.

Algumas limitações do estudo foram a não realização de um teste intra-avaliador para a medida da posição da pelve. Não foi utilizada nenhuma técnica de medida para garantir a utilização de $50 \%$ de tensão da bandagem elástica aplicada no GB, visto que a mesma foi tensionada de acordo com sua deformação.

\section{CONCLUSÃO}

Baseando-se nos resultados apresentados, a bandagem elástica auxiliou na diminuição da inclinação posterior da pelve dos participantes, podendo ser aplicada como um método auxiliar no tratamento fisioterapêutico para o alinhamento postural, apresentando maior efeito corretivo após 30 minutos e mantendo este após 72 horas. Sugere-se que sejam desenvolvidos novos estudos visando avaliar a ativação muscular e a repercussão do uso da bandagem elástica em indivíduos com inclinação posterior da pelve em associação com uma intervenção baseada na reeducação postural.

\section{AGRADECIMENTOS}

Agradecemos aos participantes pela colaboração na pesquisa. Neste estudo não houve conflito de interesses.

Todos os autores declararam não haver qualquer potencial conflito de interesses referente a este artigo.

CONTRIBUIÇÕES DOS AUTORES: Cada autor contribuiu individual e significativamente para o desenvolvimento do manuscrito. GFL (0000-0002-3782-8050** e FS (00000001-6417-4892)* foram as principais contribuintes para a redação do manuscrito. GFL, FS e GP (0000-0002-9995-9830)* realizaram a interpretação dos dados coletados e pesquisa bibliográfica. FS e GFL realizaram a revisão do manuscrito e contribuíram para o conceito intelectual do estudo. TZJ (0000-0001-8716-2333)* realizou a avaliação dos indivíduos antes e após a aplicação da bandagem, contribuindo para a coleta de dados. GFL realizou a aplicação da bandagem elástica e reuniu os dados clínicos. *ORCID (Open Researcher and Contributor ID). 


\section{REFERÊNCIAS}

1. Cho M. Correlations among pelvic positions and differences in lower extremity joint angles during walking in female university students. J Phys Ther Sci. 2015;27(6):1941-4.

2. Beach TA, Parkinson RJ, Stothart JP, Callaghan JP. Effects of prolonged sitting on the passive flexion stiffness of the in vivo lumbar spine. Spine J. 2005;5(2):145-54.

3. Polga DJ, Beaubien BP, Kallemeier PM, Schellhas KP, Lew WD, Buttermann GR, et al. Measurement of in vivo intradiscal pressure in healthy thoracic intervertebral discs. Spine (Phila Pa 1976). 2004;29(12):1320-4

4. Jaraczewska $\mathrm{E}$, Long $\mathrm{C}$. Kinesio taping in stroke: improving functional use of the upper extremity in hemiplegia. Top Stroke Rehabil. 2006;13(3):31-42.

5. Akbas E, Atay AO, Yüksel I. The effects of additional kinesio taping over exercise in the treatment of patellofemoral pain syndrome. Acta Orthop Traumatol Turc. 2011;45(5):335-41

6. Halseth T, McChesney JW, DeBeliso M, Vaughn R, Lien J. The effects of kinesio taping on proprioception at the ankle. J Sports Sci Med. 2004;3(1):1-7.

7. Santos A. Diagnóstico clínico postural: Um guia prático. 4a. ed. São Paulo: Summus; 2001

8. Faria CDCM, Lima FFP, Teixeira-SalmelaLF. Estudo da relação entre o comprimento da banda iliotibial e o desalinhamento pélvico. Rev Bras Fisioter. 2006;10(4):373-9.

9. Pinheiro L, Silva FC. Confiabilidade inter e intra-examinadores e repetibilidade baseado em um protocolo de avaliação postural [monografia]. São Paulo: Centro Universitário Metodista; 2008.

10. Bienfait M. Os desequilíbrios estáticos: fisiologia, patologia e tratamento fisioterápico. 3a. ed. São Paulo: Summus; 1995.

11. de Hoyo M, Álvarez-Mesa A, Sañudo B, Carrasco L, Domínquez S. Immediate effect of kinesio taping on muscle response in young elite soccer players. J Sport Rehabil. 2013;22(1):53-8.

12. Słupik A, Dwornik M, Białoszewski D, Zych E. Effect of Kinesio Taping on bioelectrical activity of vastus medialis muscle. Preliminary report. Ortop Traumatol Rehabil. 2007;9(6):644-51.

13. Thelen MD, Dauber JA, Stoneman PD. The clinical efficacy of kinesio tape for shoulder pain: a randomized double-blinded, clinical trial. J Orthop Sports Phys Ther. 2008;38(7):389-95.

14. Huang $C Y$, Hsieh TH, Lu SC, Su FC. Effect of the Kinesio tape to muscle activity and vertical jump performance in healthy inactive people. Biomed Eng Online. 2011;10:70.

15. Chen W, Hong W, Huang T, Hsu H. Effects of kinesio taping on the timing and ratioofvastusmedialisobliquus and vastuslateralis muscle for person with patellofemoralpain. J Biomech.2007;40(S2):318.

16. Hsu YH, Chen WY, Lin HC, Wang WT, Shih YF. The effects of taping on scapular kinematics and muscle performance in baseball players with shoulder impingement syndrome. J Electromyogr Kinesiol. 2009;19(6):1092-9.
17. Alexander CM, Stynes S, Thomas A, Lewis J, Harrison PJ. Does tape facilitate or inhibit the lower fibres of trapezius? Man Ther. 2003;8(1):37-41.

18. Cools AM, Witvrouw EE, Danneels LA, Cambier DC. Does taping influence electromyographic muscle activity in the scapular rotators in healthy shoulders? Man Ther. 2002;7(3):154-62.

19. Janwantanakul P, Gaogasigam C. Vastus lateralis and vastus medialis obliquus muscle activity during the application of inhibition and facilitation taping techniques. Clin Rehabil. 2005;19(1):12-9.

20. Fu TC, Wong AM, Pei YC, Wu KP, Chou SW, Lin YC. Effect of Kinesio taping on muscle strength in athletes-a pilot study. J Sci Med Sport. 2008;11 (2):198-201.

21. Chang HY, Chou KY, Lin JJ, Lin CF, Wang CH. Immediate effect of forearm Kinesio taping on maximal grip strength and force sense in healthy collegiate athletes. Phys Ther Sport. 2010;11(4):122-7.

22. Yoshida A, Kahanov L. The effect of kinesio taping on lower trunk range of motions. Res Sports Med 2007;15(2):103-12.

23. Mochizuki L, Amadio AC. As informações sensoriais para o controle postural. Fisioter Mov. 2006;19(2):11-18

24. Carpenter JE, Blasier RB, Pellizzon GG. The effects of muscle fatigue on shoulder joint position sense. Am J Sports Med. 1998;26(2):262-5.

25. Proske U, Wise AK, Gregory JE. The role of muscle receptors in the detection of movements. Prog Neurobiol. 2000;60(1):85-96

26. Kase K, Wallis J, Kase T. Clinical Therapeutic Applications of the Kinesio Taping Method. Tokyo, Japan: Ken Ikai Co; 2003.

27. Morrissey D. Proprioceptive shoulder taping. J Bodyw Mov Ther. 2000;4:189-94.

28. Karatas N, Bicici S, Baltaci G, Caner H. The effect of Kinesiotape application on functional performance in surgeons who have musculo-skeletal pain after performing surgery. Turk Neurosurg. 2012;22(1):83-9.

29. Lee JH, Yoo WG. The mechanical effect of anterior pelvic tilt taping on slump sitting by seated workers. Ind Health. 2011:49(4):403-9.

30. Parreira PCS, Costa LCM, Hespanhol Junior LC, Lopes AD, Costa LOP. Current evidence does not support the use of Kinesio Taping in clinical practice: a systematic review. J Physiother. 2014;60(1):31-9.

31. Kalron A, Bar-Sela S. A systematic review of the effectiveness of Kinesio Taping: fact or fashion? Eur J Phys Rehabil Med. 2013;49(5):699-709

32. Williams S, Whatman C, Hume PA, Sheerin K. Kinesio taping in treatment and prevention of sports injuries: a meta-analysis of the evidence for its effectiveness. Sports Med. 2012;42(2):153-164. 